\title{
Uma Contribuição para a Adaptabilidade de Ambi- entes Virtuais de Aprendizagem para Dispositivos Móveis
}

\author{
Viviane de F. Bartholo \\ Departamento de Informática - Universidade do \\ Norte do Paraná - Campus Luiz Meneghel, \\ Caixa Postal 261 - 86360-000 - Bandeirantes - \\ PR - Brasil \\ vbartholo@gmail.com
}

\author{
Marília A. Amaral \\ Departamento de Informática - Universidade do \\ Norte do Paraná - Campus Luiz Meneghel, \\ Caixa Postal 261 - 86360-000 - Bandeirantes - \\ PR - Brasil \\ mariliaamaral@sercomtel.com.br
}

\author{
Maria Istela Cagnin \\ Departamento de Computação e Estatística - \\ Universidade Federal do Mato Grosso do Sul \\ (UFMS), Caixa Postal 549 - 79.070-900 - \\ Campo Grande - MS - Brasil \\ istela@gmail.com
}

Resumo A popularização da computação móvel está colaborando também para o processo de ensinoaprendizagem pois, dentre outras vantagens, fornece suporte para Educação à Distância e contribui para o M-Learning (aprendizado sem hora e local pré-estabelecidos). Sob esta perspectiva, diversas ferramentas e ambientes existentes de apoio a aprendizagem, como é o caso dos Ambientes Virtuais de Aprendizagem (AVAs), devem se adequar a esta realidade. Uma solução para isso é utilizar técnicas existentes de adaptação de software, como é o caso da adaptação de conteúdo e da adaptação de interface do usuário, bem como dos diversos modelos de ambientes hipermídia adaptativos (Modelo de domínio, Modelo de usuário e Modelo de Adaptação, este último envolve o Modelo de Interface). Este artigo tem como objetivo apresentar um modelo, denominado M-AVA, para apoiar a adaptação de AVAs existentes para o contexto de dispositivos móveis, tomando como base técnicas e modelos de adaptação disponíveis na literatura. A partir da concepção do M-AVA observou-se a necessidade de incluir uma camada de adaptação na arquitetura de AVAs a fim de que os mesmos possam atender a demanda de novas tecnologias. Para mostrar a viabilidade de uso do modelo de adaptação proposto, um estudo de caso é apresentado em detalhes e novas perspectivas de pesquisa são discutidas.

Palavras-Chave: computação móvel, ambientes virtuais de aprendizagem, adaptação de conteúdo, adaptação de interface do usuário

\footnotetext{
Abstract The mobile computing popularization is also working for the teaching-learning process because, among other benefits, provides support for e-learning and contributes to the M-learning (learning without time and place pre-set). From this perspective, several existing tools and environments to support learning, such as Learning Management System (LMS), must be suitable for this. A solution to this is to use techniques of adapting existing software, such as content adaptation and the the user interface adaptation and various adaptive hypermedia environments models (domain model, user model and adaptation model). This article presents $M$-AVA model. This model support the adaptation of existing VLEs to the mobile devices context, building upon the adaptation techniques and models. From the M-AVA design there was the need to include an adaptation layer in the LMS architecture to ensure that they can meet the new technologies demands. To show the feasibility of using the proposed adaptation model, a case study is presented in detail and new research perspectives are discussed.
}

Keywords: mobile computing, learning management system, adaptation of content, adaptation of the user interface 


\section{Introdução}

O uso da Internet como ferramenta de ensino torna-se cada vez mais freqüente. O crescente desenvolvimento da tecnologia de redes de computadores com o aperfeiçoamento dos meios de comunicação, dos protocolos e das técnicas de processamento compartilhado chama a atenção para o uso de tecnologia da informação e comunicação a fim de apoiar o processo de ensino-aprendizagem [1]. A recente popularização da Internet, bem como de outros tipos de redes, está permitindo o desenvolvimento de AVAs - Ambientes Virtuais de Aprendizagem, também denominados ambientes e-learning (Aes), ou Learning Management Systems (LMS) [2].

Nos últimos anos, os AVAs se tornaram uma realidade tanto no meio acadêmico quanto no corporativo, constituindo uma alternativa tecnológica para atender ao Ensino à Distância (EAD) tanto das instituições de ensino como do meio corporativo, fornecendo uma forma diferente de apoiar o treinamento de funcionários. Com base nesta perspectiva, torna-se importante um entendimento mais crítico sobre as características que definem e orientam o uso e o desenvolvimento desses ambientes.

O uso da computação móvel e a sua difusão permitiram o desenvolvimento de aplicações móveis também na área da educação, implicando em uma nova modalidade de ensino à distância, chamada de Mobile Learning ou simplesmente M-learning [3]. Seu grande potencial encontra-se na utilização da tecnologia móvel como parte de um modelo de aprendizado integrado, caracterizado pelo uso de dispositivos de comunicação sem fio, de forma transparente e com alto grau de mobilidade [3].

Essa oferta de serviços de telecomunicações e de artefatos computacionais, capazes de prover mobilidade aos diferentes participantes de projetos educacionais, apresenta a oportunidade para o desenvolvimento de pesquisas no campo da computação móvel aplicadas à educação [4], [5]. E como migrar AVAs existentes para a nova modalidade de ensino a distância? Esta é uma das questões que este artigo procura responder.

De acordo com Pereira et al. [6], o desenvolvimento de AVAs ainda encontra-se em sua fase embrionária, muitos recursos já apontados por pesquisas tecnológicas, como agentes inteligentes e hipermídia adaptativa, continuam sendo pouco implementados em ambientes de aprendizagem, pois requerem, além de profissionais especializados, altos custos de implementação e estudos avançados de sua aplicabilidade pedagógica. Esses fatores reforçam a necessidade ao entendimento das características dos AVAs.

Dada a importância da computação móvel no meio acadêmico, o instituto SRI (Stanford Research Institute) efetuou uma pesquisa sobre o uso de dispositivos móveis nas instituições de ensino [7] apud [8] . A maioria dos professores afirmou que o uso da computação móvel proporcionou maior motivação para aprender, bem como apoiou a colaboração e a comunicação entre os estudantes uma vez que tal tecnologia contribui para facilitar o acesso ao aprendizado na obtenção de conteúdo sem hora e local pré-estabelecidos.

Ressalta-se ainda que de acordo com o relatório de pesquisa do instituto SRI no contexto brasileiro, $M$ learning ou aprendizagem com mobilidade beneficia, além do meio acadêmico, diversos setores de empresas/organizações durante as suas atividades de treinamento por meio das práticas de aprendizagem com mobilidade [9].

Diante do exposto, observa-se a importância deste artigo visto que o foco é prover um modelo, denominado M-AVA, que possibilite a adaptabilidade de AVAs para dispositivos móveis. Na Seção 2 deste artigo é apresentado o conceito de AVAs e os recursos por ele fornecido. Na Seção 3 é apresentado o conceito de aprendizagem móvel e suas classificações diante dos dispositivos móveis. Na Seção 4 apresenta-se o modelo de adaptação de AVAs para dispositivos móveis proposto, bem como as diretrizes para apoiar a sua aplicação. Na Seção 5 apresenta-se um estudo de caso conduzido para mostrar o uso do modelo proposto, que trata-se da adaptação de um AVA para celulares. Na Seção 6 são discutidas as considerações finais, contribuições e sugestões de trabalhos futuros.

\section{Ambientes Virtuais de Aprendiza- gem (AVAs)}

Segundo Kemczinski [2], os AVAs (ambientes $e$ learning) são sistemas computacionais disponíveis na Internet, destinados ao suporte de atividades de ensinoaprendizagem, mediados pelas tecnologias de informação e comunicação. Estes se apresentam integrando múltiplas mídias, linguagens e recursos tendo em vista atingir determinados objetivos de aprendizagem. As atividades desenvolvem-se no tempo, ritmo de aprendizagem e espaço em que cada participante se localiza, de acordo com uma intencionalidade explícita e um planejamento prévio.

Adicionalmente, de acordo com Santos [10], os AVAs podem ainda ser classificados como sendo uma Aplicação Hipermídia (Sistemas Hipermídia) para fornecer instrução distribuída.

De acordo com Pereira et al. [6], o número de recursos e ferramentas já desenvolvidos e em desenvolvimento para a educação baseada na Web está incentivando cada 
vez mais a utilização dos AVAs como apoio ao ensino presencial e como modalidade única de ensino aprendizagem. Isso torna cada vez mais complicado escolher, entre os recursos e as ferramentas, as que melhor ajustam-se às necessidades e aos objetivos dos programas educacionais, não existindo uma escolha correta, mas sim AVAs que se moldam melhor a determinadas necessidades. Tais recursos e ferramentas, se disponibilizados e utilizados corretamente, permitem que os participantes os utilizem para interação, colaboração e suporte do processo ensino-aprendizagem.

Com base na experiência obtida por meio do desenvolvimento de AVAs, Pereira et al. [6] afirmam que os principais recursos tecnológicos, geralmente utilizados nesses ambientes, podem ser agrupados em quatro eixos, conforme comentados a seguir e ilustrados na Figura 1.

- Documentação e informação: permitem apresentar as informações institucionais do curso, veicular conteúdos e materiais didáticos, fazer upload e download de arquivos e oferecer suporte ao uso do ambiente;

- Comunicação: facilita a comunicação síncrona e assíncrona;

- Gerenciamento pedagógico e administrativo: permite, por exemplo, acessar as avaliações e o desempenho dos aprendizes e consultar a secretaria virtual do curso

- Produção: permite o desenvolvimento de atividades e resoluções de problemas dentro do ambiente.

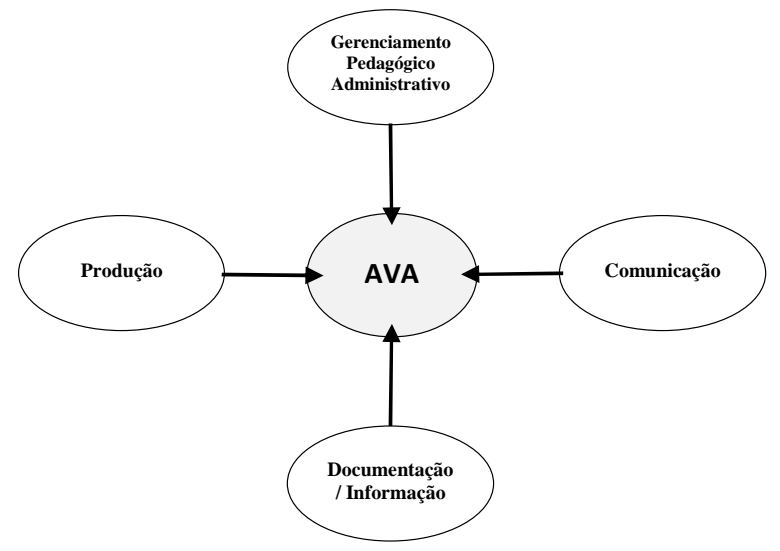

Figura 1: Eixos que definem a arquitetura de um AVA (adaptado de [6])

Vale ressaltar que de acordo com Pereira et al. [6], a falta de um destes eixos não afeta a conceituação de um AVA, pois existem diferentes tipos e modelos de AVAs. Destaca-se ainda que a quantidade de ferramentas não é fator determinante para a escolha de um AVA, mas sim a qualidade e a aplicabilidade desses ao domínio do conhecimento a ser oferecido e aos objetivos educacionais almejados.

Sob esta perspectiva, o uso de novas tecnologias, como é o caso da computação móvel pode trazer vários benefícios e auxiliar ainda mais no processo de ensinoaprendizagem.

\section{Aprendizagem Móvel e Ambientes Virtuais de Aprendizagem Móveis}

Aprendizagem Móvel (Mobile Learning - M-learning) é considerado um paradigma emergente, relacionado com três tecnologias: poder de computação do ambiente, comunicação do ambiente e desenvolvimento de interfaces inteligentes do usuário [11]. Adicionalmente, trata-se da aprendizagem que ocorre com o auxílio de dispositivos sem fios, telefones móveis, Assistentes Digitais Pessoais (PDAs) ou laptops. De acordo com as definições encontradas na literatura, Mobile Learning é o emprego de tecnologias específicas que diferenciam a aprendizagem móvel de outras aprendizagens eletrônicas (e-learning) [11].

Contudo, se considerar a mobilidade mais do ponto de vista do aluno do que da tecnologia, ela se torna mais importante, visto que a aprendizagem vai a qualquer lugar. Por exemplo, alunos fazem uma revisão para o exame no ônibus a caminho da escola, médicos atualizam seus conhecimentos médicos enquanto circulam por hospitais, estudantes de idioma melhoram habilidades de idioma enquanto viajam para outros países. Todos estes exemplos permitem o aprendizado enquanto as pessoas estão em movimento [11]. Portanto, a definição de Mobile Learning deveria ser ampliada para "qualquer tipo de aprendizagem que ocorre quando o estudante não está fixo em um local predeterminado, ou a aprendizagem que acontece quando o estudante tira vantagem das oportunidades de aprendizagem oferecidas por tecnologias móveis" [11], [5].

De acordo com Nyiri [12], o paradigma Mobile Learning surgiu aproveitando-se da disponibilidade de dispositivos móveis e considerando as necessidades específicas de educação e treinamento.

De acordo com Marçal et al. [7], as pesquisas em Mobile Learning têm se voltado para dois grupos de usuários principais, alunos ou aprendizes, que freqüentam os bancos escolares e profissionais que exercem suas atividades em campo, ou seja, externos às suas empresas. Segundo Marçal et al. [7] os dispositivos móveis fornecem um novo e motivador paradigma de interação, particularmente para as crianças, sendo que várias iniciativas têm sido desenvolvidas nesta área [5]. Já no caso dos profissionais que realizam suas atividades externas a empresa e que possuem uma rotina dinâmica, envolvendo 
viagens em diferentes locais, a preocupação está em fornecer um ambiente que coloque sempre à sua disposição a informação mais atualizada possível.

Assim, computação móvel vem propiciar uma extensão para a Educação à Distância via computadores, sendo que contribui para a facilidade de acesso ao aprendizado sem hora e local pré-estabelecidos, como já mencionado anteriormente [13], [14]. A computação móvel na educação recebe a denominação de M-learning como já visto nesta seção e pode ainda passar por diversas classificações, as quais estão apresentadas na subseção a seguir.

\subsection{Classificações de Sistemas M-learning}

Georgieva et al. [15] apresentam as diversas classificações para M-learning (Figura 2), sendo estas estabelecidas por indicadores de apoio e por normas de comunicação entre alunos e professores.

De acordo com as tecnologias da informação e comunicação, a classificação de M-learning baseia-se nos seguintes indicadores:

- tipo de suporte a dispositivos móveis: notebooks, TabletPCs, PDAs, celulares ou smatphones;

- tipo de comunicação sem fio que é utilizado para disponibilização de materiais pedagógicos e informações administrativas: GPRS (General Packet Radio Service), GSM (Global System for Mobile Communications), IEEE 802-11, Bluetooth, IrDA (Infrared Data Association).

Pode-se ainda classificar M-learning de acordo com as tecnologias educacionais baseando-se nos seguintes indicadores (Figura 2):

- suporte de comunicação: síncrona e/ou assíncrona de educação;

- apoio de normas e padrões e-learning: por exemplo SCORM (Sharable Content Object Reference Model), AICC (Aviation Industry CBT Committee);

- disponibilidade de conexão Internet permanente entre o sistema móvel da aprendizagem e os utilizadores: conexão on-line e conexão off-line;

- localização dos usuários: dentro do campus universitário (on-campus), fora do campus universitário (off-campus) e dentro e fora do campus universitário (off/on-campus);

- acesso a materiais pedagógicos e/ou serviços administrativos: sistemas M-learning com suporte ao acesso de conteúdo educativo, sistemas $M$ learning com suporte ao acesso de serviços admi- nistrativos educacionais e sistemas M-learning que suportam o acesso ao material de aprendizagem.

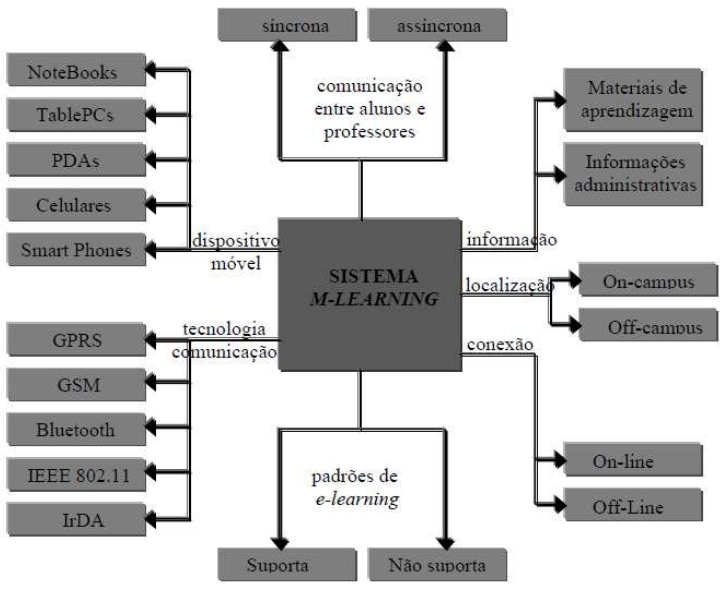

Figura 2: Classificação geral de sistemas M-learning (Extraído de [15])

A proposta de classificação oferece a possibilidade de avaliar as variedades de realizações e de requisitos para sistemas educacionais, relacionadas ao tipo de informação apoiadas e do método de acesso a elas.

O modelo de adaptação proposto neste trabalho visa permitir aos AVAs existentes apoio a todos os três tipos de aprendizagem móvel: sistemas de M-Learning com suporte a estudantes e/ou administração dos estudantes; sistemas M-Learning on-line e/ou off-line e sistemas $M$ Learning que funcione dentro e/ou fora do campus universitário.

\section{Modelo M-AVA}

Para atender as exigências dos ambientes virtuais de aprendizagem e contemplar a adaptação de tais ambientes, proporcionando adaptabilidade de AVAs para dispositivos móveis, foi proposto o modelo M-AVA. Para isso, sugere-se uma adaptação nos eixos que definem a arquitetura de um AVA, propostos inicialmente em [6], conforme apresentado na Figura 3.

A inserção da camada de "adaptação" nos eixos propostos por [6] torna o M-AVA capaz de proporcionar adaptabilidade em AVAs para dispositivos móveis. Isso ocorre por meio da definição dos elementos, tipos de adaptação e diretrizes necessárias para esta adaptação.

O modelo M-AVA toma por base inicial a definição de uma interface genérica para dispositivos móveis, ou seja, provê diretrizes para a criação de uma interface a fim de que o AVA seja utilizado por diversos tipos de dispositivos móveis, por exemplo, um celular ou PDA. Assim diversos usuários com diferentes dispositivos móveis poderão ser atendidos por um mesmo AVA. 
Uma das adaptações proposta pelo modelo M-AVA ocorrerá em tempo de construção, que é caracterizada pela construção de novas versões do AVA para diferentes tipos de dispositivos [16]. Para isso, é necessário considerar durante a fase de desenvolvimento a criação de duas aplicações, ou seja, um AVA para desktop e outro para dispositivos móveis. A outra adaptação proposta ocorrerá em tempo de execução, uma vez que dependendo do tipo de usuário que acessar a aplicação será executada uma interface diferente. Assim, o modelo M-AVA proverá a adaptação de conteúdo e a adaptação de interface do usuário.

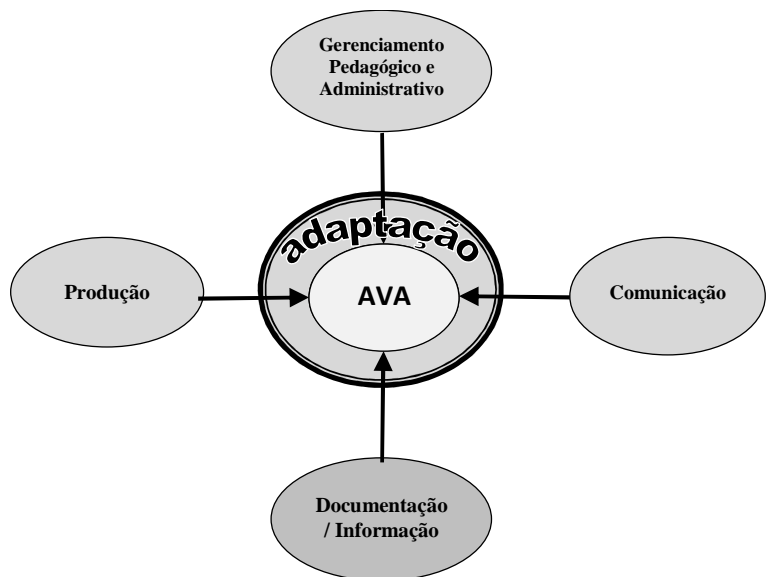

Figura 3: Eixos que definem a arquitetura de um AVA com adaptação para dispositivos móveis.

Ressalta-se que o modelo M-AVA é um modelo híbrido, tanto em relação ao tempo (a adaptação pode ocorrer em tempo de execução e em tempo de construção) quanto em relação ao tipo de adaptação (provê adaptação no contexto de conteúdo e de interface do usuário). Sendo uma vantagem em relação a outras técnicas, abordagens e modelos de adaptabilidade propostos na literatura visto que as demais se preocupam apenas com um tipo de adaptação.

No modelo proposto, todo processo de adaptação está sob a responsabilidade do servidor Web, não havendo necessidade de instalar o AVA no cliente (no caso, no desktop ou no dispositivo móvel).

Vale ressaltar que o modelo M-AVA não leva em conta aspectos pedagógicos de M-Learning, apenas os aspectos tecnológicos, uma vez que a preocupação deste trabalho é com os meios tecnológicos aos quais a aprendizagem pode utilizar para a busca do conhecimento, sem local e hora pré-estabelecidos.

A adaptação de conteúdo do modelo M-AVA é realizada no servidor de origem [17], permitindo o fornecimento de conteúdos já adaptados aos dispositivos móveis dos usuários, obrigando que o banco de dados do servidor Web (onde estarão armazenadas as informações a serem adaptadas) contenha informações de conteúdo disponível para desktop e para dispositivos móveis. Portanto, há necessidade de incluir no banco de dados campos com informações resumidas para serem melhor visualizadas no AVA móvel [17].

O modelo M-AVA considera também a adaptação de interface do usuário, tanto estática quanto dinâmica [18].

A adaptação de interface do usuário estática é alcançada durante o desenvolvimento do software pela metodologia de desenvolvimento de uma interface diferente para cada tipo de usuário, ou ainda, por meio do desenvolvimento de uma interface diferente para cada tipo de AVA (ou seja, AVAs para dispositivos móveis e AVAs para desktop). A característica estática da adaptação é obtida a partir do momento que são estabelecidas e criadas interfaces diferentes de acordo com a necessidade de cada equipamento, como por exemplo, em relação aos dispositivos móveis que é necessário selecionar as funcionalidades mais importantes dos AVAs para serem disponibilizadas devido ao tamanho da tela de cada tipo de dispositivo. A adaptação de interface do usuário estática pode também ser utilizada a partir do momento que existem vários tipos de usuários (administrador, professores e alunos) e cada um deles tem direito de acesso a um conjunto de funcionalidades do AVA.

A adaptação de interface do usuário dinâmica no modelo M-AVA é alcançada em tempo de execução do software (AVA), e considera os seguintes tipos de adaptação:

- adaptação por seleção [18]: é proporcionada pelo serviço de identificação do dispositivo. Isso foi estabelecido no modelo proposto a partir do acesso ao AVA, ou seja, dependendo do tipo de equipamento que estiver sendo utilizado (dispositivo móvel ou desktop) pelo usuário é direcionada a aplicação (AVA desktop ou AVA móvel) que deve ser acessada no servidor Web. Isto ocorre quando o usuário informa a URL (Universal Resource Locator) onde se encontra hospedado o AVA.

- adaptação pelo perfil do usuário: proporcionada pela mudança na interface de acordo com o tipo de usuário e pelas informações e funcionalidades que podem ser disponibilizadas para cada um em tempo de execução. No modelo M-AVA isso também é alcançado a partir do momento que existem vários tipos de usuários (administrador, professores e alunos) e cada um deles tem direito de acesso a um conjunto de funcionalidades do AVA.

A partir da caracterização dos elementos, ou seja, tipos de adaptação que são tratados pelo modelo proposto, recursos tecnológicos e técnicas utilizadas para obter cada tipo de adaptação que compõem o modelo M-AVA, é possível obter uma visão geral do modelo, conforme 
apresentada na Figura 4.

Tanto o AVA móvel quanto o AVA desktop estarão disponíveis no servidor Web, em conjunto com a respectiva base de dados (uma única para ambas as aplicações) a qual está adaptada para atender a adaptação baseada em conteúdo. Os usuários devem acessar o AVA independente do equipamento que possui por meio de uma URL única. Para cada AVA acessado pode ainda haver a adaptação de interface do usuário de acordo com o tipo de usuário, apresentado na Figura 4 pelos diferentes tipos de usuários possíveis (U1, U2, Un).

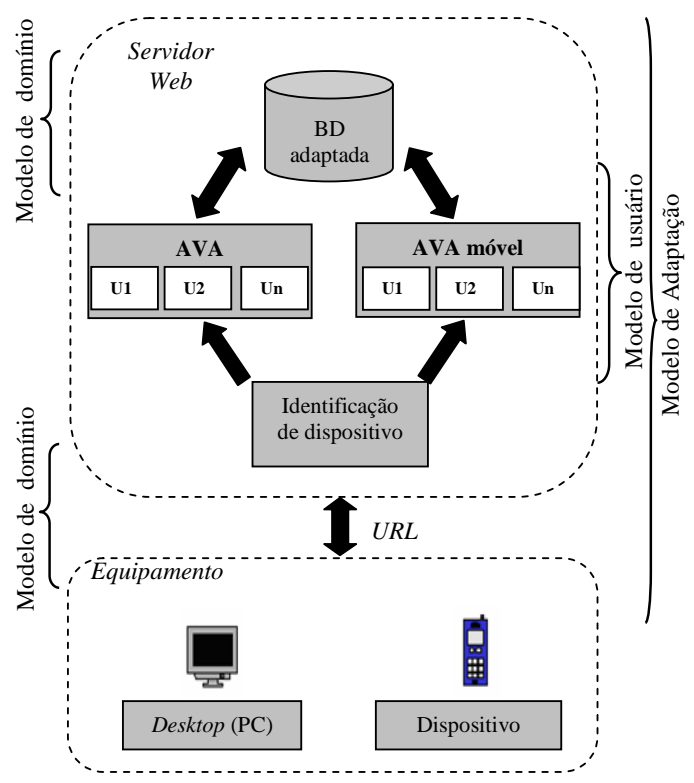

Figura 4: Modelo M-AVA

É possível notar na Figura 4 que o modelo M-AVA contempla também os modelos de domínio, de usuário e de adaptação (este último envolve o Modelo de Interface). Estes modelos, de acordo com Debra [19], estruturam a adaptabilidade de sistemas hipermídia adaptativos e ambientes hipermídia adaptativos.

O modelo de usuário é definido no M-AVA por meio de estereótipos (U1, U2, U3), com as características e necessidades de cada um dos tipos de perfil de usuário. $\mathrm{O}$ modelo de domínio é composto pelas páginas que contém as informações sobre o curso, por exemplo, e o modelo de adaptação é dado pelas regras de adaptação que definem os elementos e funcionalidades a serem apresentados aos distintos perfis de usuários. No M-AVA, os modelos de adaptabilidade de sistemas/ambientes hipermídia adaptativos são contemplados tanto pelo AVA desktop quanto pelo AVA móvel.

\subsection{Diretrizes}

Para que a adaptabilidade de AVAs para dispositivos móveis seja provida por meio do modelo M-AVA é necessário seguir alguns passos, denominados aqui como diretrizes. A sequiência das diretrizes e a indicação do tipo de adaptação, provida pelo M-AVA (descrito na Seção 4) e contemplada por cada diretriz estão ilustradas na Figura 5 por meio de um diagrama de atividades da UML (Unified Modeling Language).

A seguir tem-se uma descrição detalhada de cada diretriz a fim de facilitar o seu entendimento e aplicação. É importante salientar que as diretrizes podem ser refinadas a qualquer momento durante a adaptabilidade do AVA, desta forma, é possível aplicar novamente qualquer uma das diretrizes estabelecidas.

- Classificar AVA móvel: verificar em qual contexto de M-learning o AVA será utilizado, a fim de que se possa estabelecer como será feita a implementação da aplicação do AVA para dispositivos móveis. Sugere-se que esta classificação seja baseada na proposta de Georgieva et al. [15], apresentada na Seção 3.1.

- Identificar principais funcionalidades do AVA para dispositivos móveis: a restrição encontrada com relação a capacidade de armazenamento dos dispositivos móveis cria a necessidade de se definir quais funcionalidades do AVA desktop serão implementadas para dispositivos móveis para não sobrecarregar os dispositivos com funcionalidades desnecessárias e ao mesmo tempo facilitar o acesso. Essa diretriz atende parcialmente a adaptação de interface estática do usuário, pois são definidas as funcionalidades existentes, visando as possíveis interfaces a serem contempladas na aplicação. Ainda nesta diretriz são definidos os tipos de usuário que irão utilizar o AVA móvel, contemplando a adaptação dinâmica de interface do usuário pelo perfil.

- Adaptar o banco de dados do AVA: tanto o AVA acessado por meio de desktop como o acessado via dispositivo móvel deverão possuir o mesmo banco de dados, porém, de acordo com o modelo M-AVA alguns campos específicos deverão ser inseridos para atender aos dispositivos móveis. Desta forma é possível evitar que uma descrição de curso, por exemplo, seja exibida em texto excessivamente grande no dispositivo móvel. Esta diretriz aborda a adaptação de conteúdo no servidor.

- Identificar equipamento: a identificação do tipo de equipamento que está informando a URL de acesso ao AVA ao servidor Web é definida nesta diretriz. Caso o equipamento seja um desktop, a URL será direcionada para o acesso da aplicação 
AVA desktop. Se o equipamento for um dispositivo móvel a URL será direcionada para o AVA móvel.

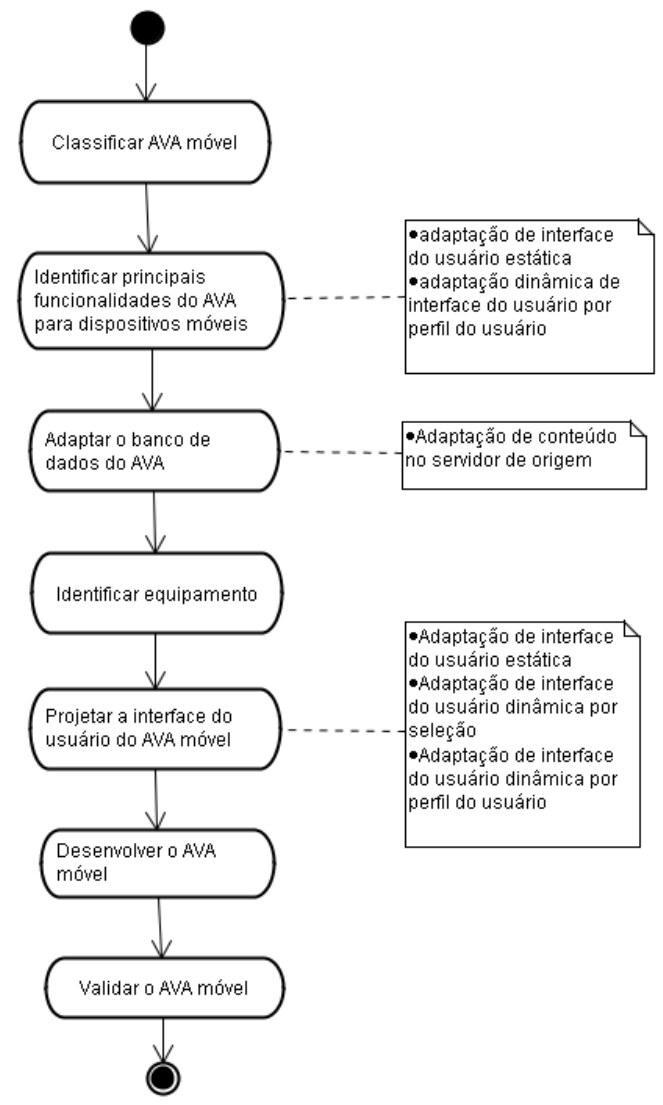

Figura 5: Fluxograma das diretrizes a serem seguidas para utilizar a arquitetura M-AVA

- Projetar a interface do usuário do AVA móvel: alguns padrões de desenvolvimento de dispositivos móveis devem ser considerados para viabilizar a adaptação de interface do usuário, tanto dinâmica como estática por seleção e por perfil do usuário. São definidas algumas subdiretrizes para um melhor desenvolvimento da adaptação de interface do usuário:

- selecionar conteúdo: manter apenas informações relevantes nas páginas, tais como: dados resumidos e funcionalidades mais adequadas ao AVA móvel. O conteúdo resumido fica adaptado no banco de dados e disponível para apresentação ao usuário, que necessitará do mínimo possível de barras de rolagem na sua navegação. Também será considerado o tamanho dos títulos de textos. Serão utilizados títulos resumidos para permanecerem menores do que os apresentados na web via desktop.
- projetar entrada de dados: as entradas de dados devem ser objetivas, curtas e de fácil digitação, pois há que se considerar as limitações presentes nos teclados.

- projetar leiaute: o leiaute das telas do AVA móvel deve primar por resoluções mínimas de telas, cores restritas e poucas informações. Porém, essas características do leiaute devem considerar os diversos tipos de dispositivos e os diferentes perfis de usuários.

- prover usabilidade: preferencialmente exibir as informações consideradas essenciais já na primeira tela do dispositivo móvel. Assim o acesso será mais rápido e as buscas por conteúdos apresentados otimizadas. $\mathrm{O}$ processo de navegação deve primar por utilização de poucos níveis.

- Desenvolver o AVA móvel: com base nestas diretrizes o desenvolvimento do AVA móvel pode ser iniciado.

- Validar o AVA móvel: a verificação do atendimento das especificações definidas nas diretrizes será realizada por meio de validação. Podem ser utilizadas técnicas de teste de software existentes na literatura, tais como teste funcional [20], para averiguar se os requisitos funcionais do aplicativo em questão foram adequados e estão coerentes, que a entrada é adequadamente aceita, que a saída esperada reflete o que foi projetado e que a integridade das informações externas é mantida, minimizando a preocupação com a estrutura lógica interna do sistema.

\section{Estudo de caso: Adaptabilidade de um AVA para celulares utilizando o modelo M-AVA}

O modelo M-AVA e sua aplicação podem ser observados em um estudo de caso desenvolvido para prover adaptabilidade em um AVA para celulares.

Para iniciar o estudo de caso, a primeira etapa contou com o desenvolvimento de um AVA desktop baseado em um documento de requisitos [21] gerado por meio de um estudo comparativo entre alguns AVAs (Moodle [22], AulaNet [23], TeleEduc [24] e WebCT [25]), a fim de verificar os serviços mais comuns oferecidos por eles.

Logo em seguida, na segunda etapa do estudo de caso, foram aplicadas as diretrizes propostas pelo modelo $\mathrm{M}$ AVA objetivando a adaptação do AVA desktop para celulares. As próximas seções apresentam a execução de cada uma das diretrizes previamente definidas durante a condução do estudo de caso. 


\subsection{Classificar AVA móvel}

A classificação do AVA móvel inicia com a definição das características de M-learning, descritas na Seção 3.1., que devem ser atendidas a fim de prover a adaptabilidade especificada no M-AVA.

$\mathrm{Na}$ definição do estudo de caso em questão, o AVA móvel foi classificado como off-line e on-line em relação a disponibilidade de conexão Internet, assim é necessário considerar dois tipos de aplicações móveis. A aplicação móvel on-line exigirá comunicação permanente entre o sistema e os usuários de dispositivos móveis, , através de um servidor web. Já a aplicação móvel off-line deve estar instalada no dispositivo móvel do usuário para o fornecimento dos materiais de aprendizagem.

Ainda foi definido que o AVA móvel não suportará especificações e normas de e-learning e sob o ponto de vista da localização do usuário, poderá ter acesso dentro e fora do campus (on-campus/off-campus) universitário.

\subsection{Identificar principais funcionalidades do AVA para dispositivos móveis}

Com o estabelecimento das características de $M$ learning presentes na aplicação móvel, foram identificadas as principais funcionalidades que o AVA móvel deve apresentar.

Considerou-se, principalmente, a relevância da funcionalidade para a sua disponibilização no AVA móvel observando as limitações de processamento destes dispositivos móveis.

O estudo de caso conta com três perfis de usuários, são eles: administrador, professor e aluno. Para cada um deles foi especificada uma funcionalidade do AVA móvel. Estas são apresentadas na Tabela 1, com suas respectivas justificativas.

\subsection{Adaptar o Banco de Dados para AVA móvel}

A especificação das funcionalidades de cada um dos perfis de usuário deu condições para a análise das tabelas do banco de dados relacionadas às mesmas. Assim foram selecionadas quais tabelas e quais campos seriam utilizados na aplicação móvel e se o conteúdo deles deveria ou não ser adaptado, considerando as dimensões e restrições de tela dos dispositivos utilizados pelos usuários.

Assim, observou-se a necessidade de adaptação de conteúdo das tabelas Cursos e Tarefas. Foi verificado que em ambas tabelas os campos relativos a descrição deveriam ser adaptados. Com isso houve a inserção de um campo denominado descricaoresumida tanto na tabela Cursos como na Tarefas.

Tabela 1.: Funcionalidades do AVA móvel

\begin{tabular}{|c|c|}
\hline $\begin{array}{c}\text { Nome da } \\
\text { Funcionalidade }\end{array}$ & Justificativa \\
\hline $\begin{array}{l}\text { F1. o administrador } \\
\text { poderá cadastrar um } \\
\text { usuário remotamente }\end{array}$ & $\begin{array}{l}\text { Funcionalidade relevante } \\
\text { com pouco poder de pro- } \\
\text { cessamento, uma vez que } \\
\text { as informações necessá- } \\
\text { rias para o cadastro são } \\
\text { do tipo texto e curtas. }\end{array}$ \\
\hline $\begin{array}{l}\text { F2. O administrador } \\
\text { poderá enviar e-mail } \\
\text { para um ou todos os } \\
\text { níveis de usuário do } \\
\text { AVA desktop }\end{array}$ & $\begin{array}{l}\text { Funcionalidade relevante } \\
\text { ao usuário administrador } \\
\text { que deve manter contato } \\
\text { com os demais tipos de } \\
\text { usuários com facilidade. }\end{array}$ \\
\hline $\begin{array}{l}\text { F3. O professor pode- } \\
\text { rá visualizar todos } \\
\text { os cursos que minis- } \\
\text { tra }\end{array}$ & $\begin{array}{l}\text { Funcionalidade relevante } \\
\text { e importante para o usuá- } \\
\text { rio professor ter contro- } \\
\text { le sobre os cursos que } \\
\text { ministra. }\end{array}$ \\
\hline $\begin{array}{l}\text { F4. O professor pode- } \\
\text { rá cadastrar uma nova } \\
\text { tarefa remotamente }\end{array}$ & $\begin{array}{l}\text { Funcionalidade relevante } \\
\text { e importante para o usuá- } \\
\text { rio professor poder dis- } \\
\text { ponibilizar a qualquer } \\
\text { momento e de qualquer } \\
\text { lugar tarefas aos alunos } \\
\text { cadastrados em seu(s) } \\
\text { curso(s). }\end{array}$ \\
\hline $\begin{array}{l}\text { F5. O aluno poderá } \\
\text { visualizar todos os } \\
\text { cursos em que está } \\
\text { matriculado }\end{array}$ & $\begin{array}{l}\text { Funcionalidade relevante } \\
\text { e importante para o aluno } \\
\text { possa manter um controle } \\
\text { dos cursos em que ele se } \\
\text { encontra cadastrado. }\end{array}$ \\
\hline $\begin{array}{l}\text { F6. O aluno poderá } \\
\text { visualizar todas as } \\
\text { tarefas de um deter- } \\
\text { minado curso remota- } \\
\text { mente }\end{array}$ & $\begin{array}{l}\text { Funcionalidade relevante } \\
\text { e importante para o aluno } \\
\text { possa acompanhar constan- } \\
\text { temente suas tarefas a } \\
\text { serem executadas sem hora } \\
\text { e local determinado. }\end{array}$ \\
\hline
\end{tabular}

\subsection{Identificar equipamento}

Sabendo dos meios para estabelecer a adaptação de conteúdo, deve-se identificar o tipo de equipamento que está acessando a URL do AVA no servidor Web. Entre os tipos de equipamentos tem-se: desktop ou dispositivo móvel, esse último podendo ser classificado em: celular, palm, smartphone, dentre outros.

Após estabelecer a adaptação de conteúdo, é necessário identificar o tipo de equipamento (desktop ou dispositivo móvel - celular, palm, smartphone, dentre outros) que está acessando a URL do AVA no servidor Web.

A adaptação em tempo de execução é a base para a implementação desta diretriz, pois de acordo com o tipo de equipamento (desktop ou dispositivo móvel) o acesso é direcionado para um tipo de aplicação, AVA desktop ou 


\section{AVA móvel.}

Assim, para prover a adaptação em tempo de execução nesta diretriz verifica-se se o equipamento é um desktop então a aplicação é direcionada para o acesso a aplicação AVA desktop, por outro lado, se o equipamento é um dispositivo móvel então o acesso é direcionado para a aplicação AVA móvel.

A tecnologia de user-agent [16] foi escolhida para tratar a identificação do equipamento. Tal escolha se deu pelo fato de ser a mais comum e a mais simples de ser utilizada. Neste estudo de caso, foi verificado, especificamente, o tipo de sistema operacional (sistema operacional para desktop ou para dispositivo móvel) que o equipamento de origem de acesso à URL possui a fim de direcionar à aplicação correta. É importante ressaltar que o objetivo é identificar o tipo de dispositivo móvel de forma generalizada, ou seja, se é um desktop ou um dispositivo móvel (celular, PDA, etc).

\subsection{Projetar a interface do usuário do AVA móvel}

O projeto da interface do usuário do AVA móvel considerou alguns padrões de desenvolvimento, abordados pelas subdiretrizes do M-AVA, e estão detalhados a seguir:

- selecionar conteúdo: essa subdiretriz foi aplicada no estudo de caso uma vez que procurou-se: a) estabelecer os textos de entrada com tamanho o mais curto possível e b) considerar como conteúdo dos textos das saída os campos resumidos, resultantes da adaptação de conteúdo executada na diretriz "Adaptar banco de dados do AVA móvel".

- projetar entrada de dados: para o estudo de caso minimizou-se as funcionalidades que de uma maneira ou outra se apropriavam de atividades de entrada de dados para o AVA móvel. Assim o foco foi a visualização da informação.

- projetar leiaute: as cores utilizadas no leiaute foram preto e branco (e suas variações em escala de cinza) possibilitando assim o uso do AVA móvel em diversos tipos de celulares, inclusive nos que não podem oferecer recurso de telas com resolução colorida. Primou-se por uma interface simples, para evitar dificuldade de uso e acesso.

- prover usabilidade: como todas as subdiretrizes anteriores foram cumpridas, então essa pode ser considerada aplicada também. Assim todas elas contribuem para que as informações sejam claras, curtas, objetivas, precisas e de fácil acesso para o usuário.

\subsection{Desenvolver o AVA móvel}

Após aplicar todas as diretrizes anteriores é necessário efetuar o desenvolvimento do AVA móvel propriamente dito. Para prover a adaptabilidade desejada para o AVA desktop foram desenvolvidos dois tipos de aplicações móveis (AVA móvel): off-line e on-line.

No desenvolvimento da aplicação móvel on-line (Figura 6a) foram utilizadas as tecnologias WAP (Wireless Application Protocol), WML (Wireless Markup Language) e JSP (JavaServer Pages). A ferramenta de desenvolvimento utilizada foi o NetBeans [26] e o emulador de celular utilizado foi o EasyPad WAPtor [27].

No desenvolvimento da aplicação móvel off-line (Figura 6b) foi utilizada a tecnologia J2ME (Java Micro Edition). A ferramenta de desenvolvimento utilizada foi o NetBeans [26] e o emulador de celular utilizado foi o Wireless ToolKit [28]. A comunicação AVA móvel offline com o servidor Web para ter acesso ao banco de dados é realizada por meio do protocolo HTTP (HyperText Transfer Protocol), que é implementado na especificação J2ME pela interface HttpConnection.
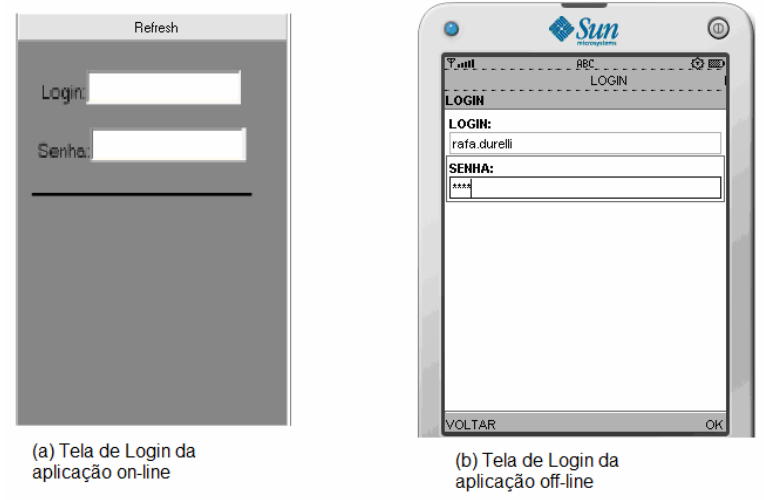

Figura 6: Telas de Login das aplicações on-line e off-line

\subsection{Validar o AVA móvel}

Inicialmente, verificou-se que todas as especificações definidas nas diretrizes do modelo M-AVA foram atendidas pelas aplicações móveis on-line e off-line.

Por exemplo, em relação as aplicações on-line e offline foi verificado se a adaptação em relação ao banco de dados e se a identificação do equipamento que está acessando as aplicações foram feitas de maneira correta.

Para validar a adaptação do banco de dados, observou-se: 1) se as funcionalidades mais importantes do AVA estavam presentes na aplicação AVA móvel e quais eram os dados importantes dessas funcionalidades; e 2) se foi criado um campo resumido no banco de dados para cada conteúdo extenso de cada informação importante, 
presente nas funcionalidades disponíveis no AVA móvel.

Para validar a identificação do equipamento que está acessando a aplicação (móvel ou desktop) observou-se se o servidor Web direcionou o acesso para o tipo de aplicação correta (AVA desktop ou AVA móvel), de acordo com o tipo de equipamento (desktop ou dispositivo móvel) que enviou a URL do AVA para tal servidor.

Em seguida, verificou-se se todas as funcionalidades identificadas na diretriz "Identificar principais funcionalidades do AVA para dispositivos móveis" estavam presentes tanto na aplicação móvel on-line quanto na off-line e se estavam funcionando corretamente. Para facilitar esta análise, a especificação de tais funcionalidades, disponível no documento de requisitos, foi utilizada.

Salienta-se que a idéia geral de teste funcional foi tomada como base, no entanto, nenhum critério de teste funcional específico foi utilizado.

\section{Conclusões}

Na prática do estudo de caso o Modelo de Interface do M-AVA ficou responsável pela adaptação em tempo de construção, que é caracterizada pela construção de novas versões do AVA para diferentes tipos de dispositivos. Essa adaptação considerou os três perfis de usuários definidos no estudo de caso, professor, aluno e administrador. A adaptação de interface pode ser efetivada tanto de forma estática como dinâmica, esta última pode ser por seleção ou por perfil do usuário.

O Modelo de Usuário, definido no estudo de caso do M-AVA, interfere na adaptação em tempo de execução, uma vez que dependendo do tipo de usuário que acessar a aplicação será executada uma interface diferente.

No estudo de caso apresentado neste artigo, verificouse que a adaptação de conteúdo deveria ser estabelecida nas tabelas Cursos e Tarefas. Essa adaptação de conteúdo está relacionada com o Modelo de Domínio. Notou-se que nas tabelas Cursos e Tarefas os campos relativos às respectivas descrições foram adaptados com a inserção do campo descriçãoresumida tanto na tabela Cursos quanto na tabela Tarefas.

O modelo M-AVA contribui tanto para a área de Educação quanto para a área de Treinamento Empresarial, que atualmente utiliza cada vez mais m-learning. Adicionalmente, fornece contribuição para a área de Engenharia de Software pois provê diretrizes de adaptação de um sistema de domínio específico, no caso AVAs, para o contexto de dispositivos móveis, que é uma área em crescimento atualmente.

Assim, o desenvolvimento deste trabalho colaborou para a linha de pesquisa relacionada a adaptabilidade de ambientes virtuais de aprendizagem para dispositivos móveis, oferecendo uma nova alternativa para o desenvolvimento de uma aplicação que possa abranger diversos tipos de dispositivos, identificando principalmente novos campos de estudo em direção a novas formas de implementação de acordo com a classificação de $m$ learning escolhido, além da contribuição para um maior desenvolvimento de estudos na área de m-learning, cada vez mais necessária nos dias de hoje.

Este trabalho abre ainda uma série de questões que podem ser exploradas como trabalhos futuros de pesquisa, como agregar aspectos pedagógicos de m-learning ao modelo M-AVA e identificar outros tipos de adaptação que podem ser adicionados ao modelo proposto para que a mesma possa ser aplicada não somente no contexto de adaptabilidade de AVAs.

\section{Referências}

[1] L.M.M. Giraffa. Uma Arquitetura de Tutor Utilizando Estados Mentais. Tese Doutorado em Ciências da Computação - Instituto de Informática, UFRGS, Porto Alegre, 166 p., 1999.

[2] A. Kemczinski. Método de Avaliação para Ambientes E-Learning. Tese Doutorado em Engenharia da Produção da UFSC - Universidade Federal de Santa Catarina, Florianópolis-SC, 2005.

[3] M. Scopel. WSMEL: uma arquitetura para integração de serviços educacionais usando dispositivos móveis na formação de comunidades virtuais espontâneas. Dissertação de mestrado. Universidade do Vale do Rio dos Sinos, Programa de PósGraduação em Computação Aplicada,109 p., 2005.

[4] L. Meirelles; L. Tarouco; C. Alves. Telemática Aplicada à Aprendizagem com Mobilidade. RENOTE - Revista Novas Tecnologias na Educação. CINTED-UFRGS. v.2, n. 2, Novembro, 2004. Novas, Porto Alegre, 2004.

[5] D. Levis; J. Barbosa; S. Pinto, D. Barbosa. Aperfeiçoamento Automático do Perfil do Aprendiz em Ambientes de Educação Ubíqua. In: Revista Brasileira de Informática na Educação, Volume 16 - Número 1, 2008. URL: http://bibliotecadigital.sbc.org.br/download.php?p aper=1057, último acesso em julho/2009. 
[6] A.T.C. Pereira; V. Schmitt; M.R. A. C. Dias. Ambientes Virtuais de Aprendizagem. In: Pereira, A.T.C. (Org), AVA -Ambientes Virtuais de Aprendizagem em diferentes contextos. 1. ed. Rio de Janeiro: Editora Ciência Moderna. 2007. Cap. 1, p. 4-22.

[7] E. Marçal; R. Andrade; R. Rios. Aprendizagem utilizando Dispositivos Móveis com Sistemas de Realidade Virtual. RENOTE - Revista Novas Tecnologias na Educação, CINTED-UFRGS . v. 3, n.1, Maio, 2005. Porto Alegre, 2005.

[8] V. Crawford; P. Vahey. Palm Education Pioneers Program. March, 2002. Evaluation Report. SRI International, Estados Unidos.

[9] N. Reinhard; A. Z. Saccol; E. Schlemmer, J.L.V. Barbosa; S. Kristoffersen. M-learning ou Aprendizagem com Mobilidade e sua utilização no contexto Brasileiro, RELATÓRIO DE PESQUISA 01/2007. Projeto de pesquisa: Aprendizagem com Mobilidade no contexto organizacional. Edital MCT/CNPq 02/2006.

[10] N. Santos. Estado da arte em espaços virtuais de ensino e aprendizagem., Revista Brasileira de Informática na Educação, s.1., n.4,p.75-94, abr. 1999.

[11] D. Dochev; I. Hristov. Mobile Learning Applications Ubiquitous Characteristics and Technological Solutions. Bulgarian Academy Of Sciences Cybernetics And Information Technologies, vol. 6, no 3, Sofia, 2006

[12] K. Nyiri. Towards a Philosophy of M-learning. This paper appears in: Wireless and Mobile Technologies in Education, 2002. Proceedings. IEEE International Workshop on, p. 121- 124, ISBN: 07695-1706-4, 2002.

[13] A. Trifonova; E. Georgieva; M. Ronchetti (2006). Determining Students' Readiness for Mobile Learning. In: Proceedings of the The $5^{\text {th }}$ WSEAS International Conference on E-ACTIVITIES (ELearning, E-Communities, E-Commerce, EManagement, E-Marketing, E-Governance, TeleWorking) (E-ACTIVITIES '06), 2006, Venice, Italy. (Extended version published in the WSEAS Transactions on Advances in Engineering Education).

[14] C. Chang; J. Sheu (2002). Design and Implementation of Ad Hoc Classroom and eSchoolbag Systems for Ubiquitous Learning. IEEE International Workshop on Wireless and Mobile Technologies in Education, 2002, Växjö, Sweden. p. 814
[15] E. Georgieva; A. Smrikarov; T. Georgiev. A General Classification of Mobile Learning Systems. International Conference on Computer Systems and Technologies - CompSysTech' 2005.

[16] W.V. Carvalho. Um Ambiente de Desenvolvimento de Aplicações Multi-Plataformas e Adaptativas para Dispositivos Móveis. Dissertação de Mestrado em Ciência da Computação. Universidade Federal do Ceará. Fortaleza - CE, 118 p. 2005.

[17] L.H. Santana; D.S. Martins; C.B. Do Perlin; A.F. Prado; W.L. Souza; M. Biajiz. Adaptação de Páginas Web para Dispositivos Móveis. In: WebMedia'07, Outubro 2007, Gramado-RS, 2007.

[18] G. Menkhaus. Adaptive User Interface Generation in a Mobile Computing Environment. PhD Thesis, Department of Computer Science, University of Salzburg, Austria 2002. URL: www.softwareresearch.net/site/publications/, último acesso em agosto/2008.

[19] P. De Bra; Ad. Aerts; G. Houben; H. Wu,. AHAM: A Dexter-based Reference Model for Adaptive Hypermedia. In: ACM Conference on Hypertext andHypermedia, 10, Darmstadt, Germany, 1999, p.147-156.

[20] R.S. Pressman. Engenharia de Software, 6 ed., Rio de Janeiro: Mc Garw Hill, 2006.

[21] V. Bartholo. M-AVA: Arquitetura para apoiar a Adaptabilidade de Ambientes Virtuais de Aprendizagem para Dispositivos Móveis, Dissertação apresentada ao Programa de Mestrado em Ciência da Computação do Centro Universitário Eurípides de Marília, Fundação de Ensino "Eurípides Soares da Rocha”, Marília, São Paulo, 2009.

[22] Moodle. URL: http://moodle.org, último acesso em agosto/2009.

[23] Aulanet. URL: http://www.eduweb.com.br/, último acesso em agosto/2009.

[24] Teleduc. URL: http://www.teleduc.org.br/, último acesso em agosto/2009.

[25] WebCT. URL: www.webct.com, último acesso em agosto/2008.

[26] NetBeans IDE. URL: www.netbeans.org, último acesso em setembro/2008.

[27] WapTop Net. EasyPad WAPtor URL: www.waptop.net, último acesso em setembro/2008. 
[28] Java Sun. Wirelles Toolkit. URL: java.sun.com/products/sjwtoolkit, último acesso em setembro/2008. 